Research Article

\title{
The Cataleptic, Asymmetric, Analgesic, and Brain Biochemical Effects of Parkinson's Disease Can Be Affected by Toxoplasma gondii Infection
}

\author{
Mahnaz Taherianfard $\left(\mathbb{D},{ }^{1}\right.$ Moslem Riyahi, ${ }^{1}$ Mostafa Razavi, ${ }^{2}$ Zahedeh Bavandi, \\ Narges Eskandari Roozbahani, ${ }^{3}$ and Mohammad Mehdi Namavari ${ }^{4}$ \\ ${ }^{1}$ Physiology Division, Department of Basic Science, School of Veterinary Medicine, Shiraz University, Shiraz, Iran \\ ${ }^{2}$ Department of Pathobiology, School of Veterinary Medicine, Shiraz University, Shiraz, Iran \\ ${ }^{3}$ Pharmacology Division, Department of Basic Sciences, School of Veterinary Medicine, Shiraz University, Shiraz, Iran \\ ${ }^{4}$ Razi Vaccine and Serum Research Institute, Shiraz, Iran
}

Correspondence should be addressed to Mahnaz Taherianfard; taherian@shirazu.ac.ir

Received 1 September 2019; Accepted 18 March 2020; Published 5 May 2020

Guest Editor: Moustafa Gabr

Copyright (c) 2020 Mahnaz Taherianfard et al. This is an open access article distributed under the Creative Commons Attribution License, which permits unrestricted use, distribution, and reproduction in any medium, provided the original work is properly cited.

\begin{abstract}
Purpose. Parkinson's disease (PD) is a neurodegenerative disorder with progressive motor defects. Therefore, the aim of the present investigation was to examine whether catalepsy, asymmetry, and nociceptive behaviors; the Nissl-body and neuron distribution; brain-derived neurotrophic factor (BDNF); malondialdehyde (MDA); total antioxidant capacity (TAC) levels; and the percentage of dopamine depletion of striatal neurons in the rat model of Parkinson's disease (PD) can be affected by Toxoplasma gondii (TG) infection. Methods. Fifty rats were divided into five groups: control (intact rats), sham (rats which received an intrastriatal injection of artificial cerebrospinal fluid (ACSF)), PD control (induction of PD without TG infection), TG control (rats infected by TG without PD induction), and PD infected (third week after PD induction, infection by TG was done). PD was induced by the unilateral intrastriatal microinjection of 6-hydroxydopamine (6-OHDA) and ELISA quantified dopamine, BDNF, MDA, and TAC in the striatum tissue. Cataleptic, asymmetrical, nociceptive, and histological alterations were determined by bar test, elevated body swing test, formalin test, and Nissl-body and neuron counting in the striatal neurons. Results. The results demonstrated that PD could significantly increase the number of biased swings, descent latency time, and nociceptive behavior and decrease the distribution of Nissl-stained neurons compared to the control and sham groups. TG infection significantly improved biased swing, descent latency time, nociceptive behavior, and the Nissl-body distribution in striatal neurons in comparison to the PD control group. The striatal level of BDNF in the PD-infected and TG control groups significantly increased relative to the PD control group. The striatal MDA was significantly higher in the PD control than other groups, while striatal TAC was significantly lower in the PD control than other groups. Conclusions. The current study indicates that TG infection could improve the cataleptic, asymmetric, nociceptive and behaviors; the level of striatal dopamine release; BDNF levels; TAC; and MDA in PD rats.
\end{abstract}

\section{Introduction}

Parkinson's disease (PD) is one of the most widespread neurodegenerative diseases, with a prevalence of approximately $1 \%$ of individuals with an age above 65 years [1]. It determined by the chronic and slowly progressive injury and depletion of dopaminergic neurons in the substantia nigra that may led to motor disturbances, including bradykinesia, akinesia, rest tremor, and postural instability. Likewise, patients may suffer from nonmotor symptoms, including depression, anxiety, rapid eye movement (REM) disorder, and pain $[2,3]$. Catalepsy, the impairment of movement initiation, is an extrapyramidal dysfunction known as a prominent motor symptom of $\mathrm{PD}$, which is related to the striatal 
dopamine reduction. Cataleptic behavior in the PD rodent has been employed as a standard model of bradykinesia and rigidity in human PD [4]. Pain is an important nonmotor symptom in $\mathrm{PD}$ patients that overshadow the quality of PD's life. Forty to 85 percentages of PD patients have painful sensations [3] in five different manners: musculoskeletal (related to parkinsonian rigidity and akinesia), neuropathic (related to neural lesion), dystonia-related, akathisia, and central neuropathic pain [5]. Although some genetic mutations identified have been associated with $\mathrm{PD}$, the exact causes of PD remain unknown. Nevertheless, the crucial roles of oxidative stress and neuroinflammation in its pathogenesis have been well documented [6]. Even though the underlying cause of idiopathic PD remains unknown, the role of an increase in the oxidative stress, inflammatory responses, and mitochondrial dysfunction of dopamine neurons and a decrease in the availability of brain-derived neurotrophic factor (BDNF) have been well documented as pathophysiologic mechanisms [7].

Toxoplasma gondii (TG), a member of the phylum Apicomplexa, is an obligate intracellular parasite with high worldwide distribution which has infected one-third of the world population [8]. Tachyzoites, the invasive form of TG, infect neural cells. Afterward, in rodents, they differentiate into bradyzoites, which form the intracellular brain cysts. TG infection is considered asymptomatic in adults, but it can cause problems such as encephalitis, blindness, and mental retardation in immunocompromised individuals and congenitally infected children [8]. It has been observed that brain biochemistry is affected by the host immune system responses against TG infection [9]. On the other hand, TG protects itself and host cells against the immune response through some strategies, such as increasing the levels of antioxidant activity [10], brain-derived neurotrophic factor )BDNF( [11], dopamine concentration [12], and antiapoptotic activity [13]. IFN- $\gamma$ production is increased by TG infection through tyrosine hydroxylase activity 1 (TH1) immune response [14]. Torres et al. found that TG reduces the expression of N-methyl-D-aspartate (NMDA) receptor via IFN- $\gamma$ stimulation [15]. Moreover, TG activates the astrocytes that synthesize some metabolites to modulate NMDA receptor function [16]. The dopamine levels can be changed by TG in the brain [12]. The behavioral changes observed in infected rodents are attributed to its ability to change the neurotransmitter levels [12]. Dopamine depletion in PD decreases the inhibitory control of the corticostriatal glutamatergic pathway [17]. Moreover, PD causes hypofunction of the output pathways from the substantia nigra and medial globus pallidus, which leads to the increase in the excitatory subthalamic glutamatergic output [18].

Striatal medium spiny neurons contained dopamine D2 receptors, which are involved in controlling extrapyramidal functions. Haloperidol, an antipsychotic agent, induces catalepsy via selective blocking of dopamine D2 receptor in the striatum [19]. Previous findings indicated that the dopamine system has a modulatory effect on the pain and dopamine D2 receptor agonists attenuate nociceptive behavior initiation in animals and humans. Therefore, striatal dopamine enhancement can attenuate PD pain and catalepsy symptoms. There are a few investigations on the interaction of TG infection with PD pain symptom and behavioral changes; and these results are in paradox; also, most of the study on the relation between TG and PD were done according to a serological study in human and there is not any study on the brain. Therefore, the present investigation was done to study the effects of TG infection on experimental PD induction rat's asymmetric and cataleptic behavior, pain perception, percentage of dopamine depletion, level of striatal BDNF, TAC and MDA levels, and Nissl-body and neuron distribution in striatal neurons.

\section{Materials and Methods}

2.1. Subjects and Study Design. Fifty Sprague Dawley male rats, weighing 220-300 g in standard condition 12-h light/dark cycle with food and water ad libitum were used. Animal handling was conducted according to the Ethical Committee for Animal Experiments at Shiraz University. Animals were randomly divided into five groups ( $n=10$ in each group): control group, intact rats; sham group, intrastriatally ACSFinjected rats; $\mathrm{PD}$ control group, induction of $\mathrm{PD}$ in rats by intrastriatal injection of 6OHDA; TG control group, rats were infected by TG; and PD-infected group, the infection by TG was done on the third week after the induction of PD..

RH strain TG tachyzoites were obtained from the Razi Vaccine and Serum Research Institute, Shiraz, Iran. For infection induction, rats received intraperitoneal (IP) injection of $200 \mu \mathrm{l}$ normal saline containing $10^{5}$ tachyzoites (counted by a hemocytometer). At the end of the experiment, for the confirmation of tissue cyst formation, the brain smears were prepared and stained by Giemsa (Figure 1(a)).

2.2. Behavioral Testing. Rats were subjected to elevated body swing test by the method of Roghani et al. [20]. Catalepsy was evaluated by the bar test. In brief, the forepaws of each rat were located in a half-rearing position on a horizontal metal bar, which was set at $9 \mathrm{~cm}$ above the base in a parallel form. The time was recorded until the rats removed one paw from the bar (descent latency time). Descent latency cutoff time was $180 \mathrm{sec}$ for the bar test [21]. The PD-infected design of the behavioral tests was performed in four steps: first, one week before the surgery; second and third, two and three weeks after surgery, respectively; and forth, eight weeks after TG infection (Figure 1(b)).

2.3. Formalin Test. Pain behavior was examined by the formalin test eight weeks after the infection as follows: the rats were located in the $32 \times 32 \times 32 \mathrm{~cm}$ Plexiglas boxes and a mirror was mounted at a $45^{\prime \prime}$ angle, beneath the floor to the view of the rats' paws. $50 \mu \mathrm{l}$ of $2.5 \%$ formalin was injected by $27 \mathrm{G}$ needle into the foot plantar surface of the hind paw contralateral to the 6-OHDA injection site. The pain behavior was scored in $15 \mathrm{sec}$ intervals and continued for $60 \mathrm{~min}$ [22]. After behavioral testing, the animals were decapitated and the striatum was dissected and stored at $-80 \mathrm{C}^{\circ}$.

2.4. Stereotaxic Surgery. Animal was anesthetized by IP injection of ketamine $(100 \mathrm{mg} / \mathrm{kg})$ and xylazine $(8 \mathrm{mg} / \mathrm{kg}) . \mathrm{PD}$ induction was done by a unilateral and single injection of 


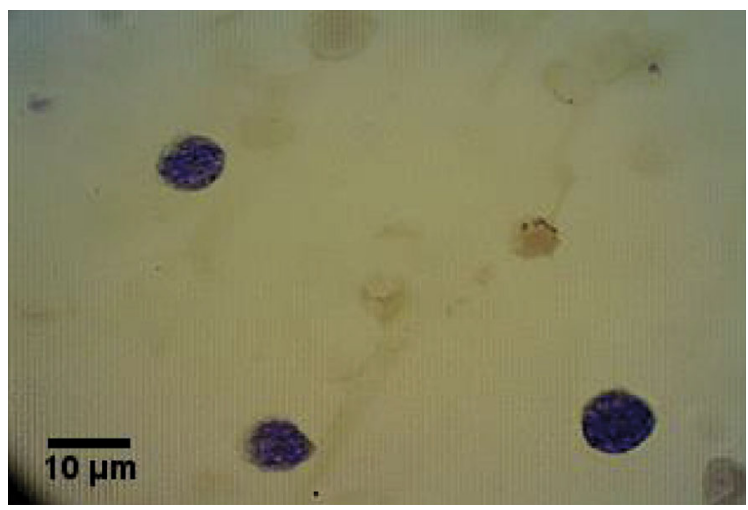

(a)

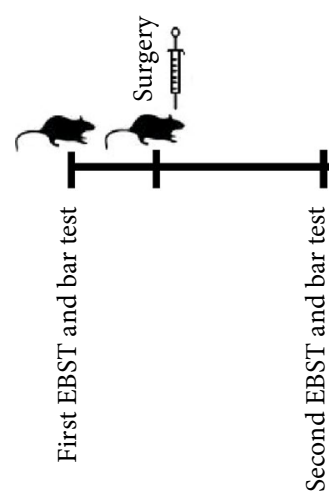

Figure 1: (a) Photomicrograph showing Toxoplasma gondii cysts (Giemsa stained) in a brain smear of infected rats. (b) The overall design of the study and the scheduling of behavioral tests. (c) Photomicrograph shows the rat brain section in the striatum region confirming the injection site.

$20 \mu \mathrm{g}$ of 6-OHDA (in $4 \mu \mathrm{l}$ saline with $0.2 \mathrm{mg} / \mathrm{ml}$ of ascorbic acid) into the striatum $(0.7 \mathrm{~mm}$ anterior to the bregma, $3 \mathrm{~mm}$ lateral to the midline, and $5 \mathrm{~mm}$ ventral to the dura).

The accuracy of the injection site location was determined by the microinjection of $4 \mu \mathrm{l}$ methylene blue into the striatum at the end of each experiment. In decapitated rats, the brains were removed and then were fixed in $10 \%$ of phosphate-buffered formalin for 24 hours. The striatum injection site was confirmed by comparing it with the atlas of Paxinos and Watson (Figure 1(c)).

2.5. Nissl Body and Neuron Distribution in the Striatum. At the end of the experiments, the rats were deeply anesthetized and sacrificed. For Nissl-body and neuron distribution measuring, after brain tissue fixation by $10 \%$ formalin, automatic tissue processor prepared paraffin blocks; then, $5 \mu$ coronal section of the brain blocks in the region of striatum neurons was prepared. In all sections, Nissl-body counting in six cells and cell counting in six parts of each slide section of the striatum in all of the groups by Cresyl violet and hematoxylin eosin staining were done.

2.6. Dopamine, BDNF, TAC, and MDA Measurement. Dopamine concentration was measured using an ELISA kit (RE59161, IBL, Hamburg, Germany) in $50 \mu \mathrm{g}$ of homogenized striatal tissues based on Mabandla et al.'s modification
[23]. The percentage of dopamine relative to the control group was determined according to following formula:

$$
\frac{\text { dopamine concentration of group } x}{\text { dopamine concentration of control group }} \times 100
$$

A rat ELISA kit (MyBioSource, USA, catalog \# MBS824814) according to the manufacturer's instructions measured striatal BDNF. An ELISA standard kit was used to evaluate striatal TAC (ZB-TAC-96A, Zell Bio Germany) and MDA (ZB-MDA-96A, Zell Bio Germany).

2.7. Statistical Analysis. SPSS version 22 was used for data analysis. The Kolmogorov-Smirnov test was used for verifying the normalization of data. One-way ANOVA and Tukey's test, as the post hoc test, were used, and the significant level was considered $P<0.05$.

\section{Result}

Catalepsy, via the bar test, revealed a significant increase in the descent latency time at the second, third, and fourth steps in the PD control group compared to the control and sham groups $(P<0.001)$. In addition, the $\mathrm{PD}$-infected group showed a significant $(P<0.001)$ increase in the descent latency time at the second and third steps, but not at the 


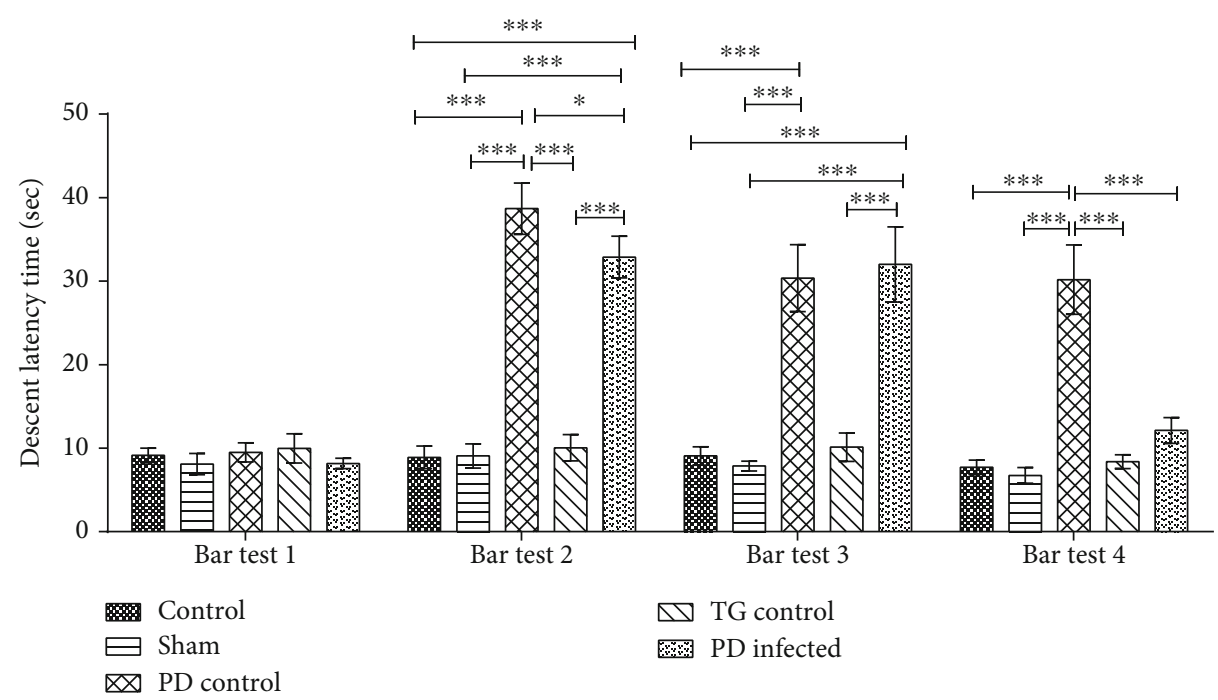

(a)

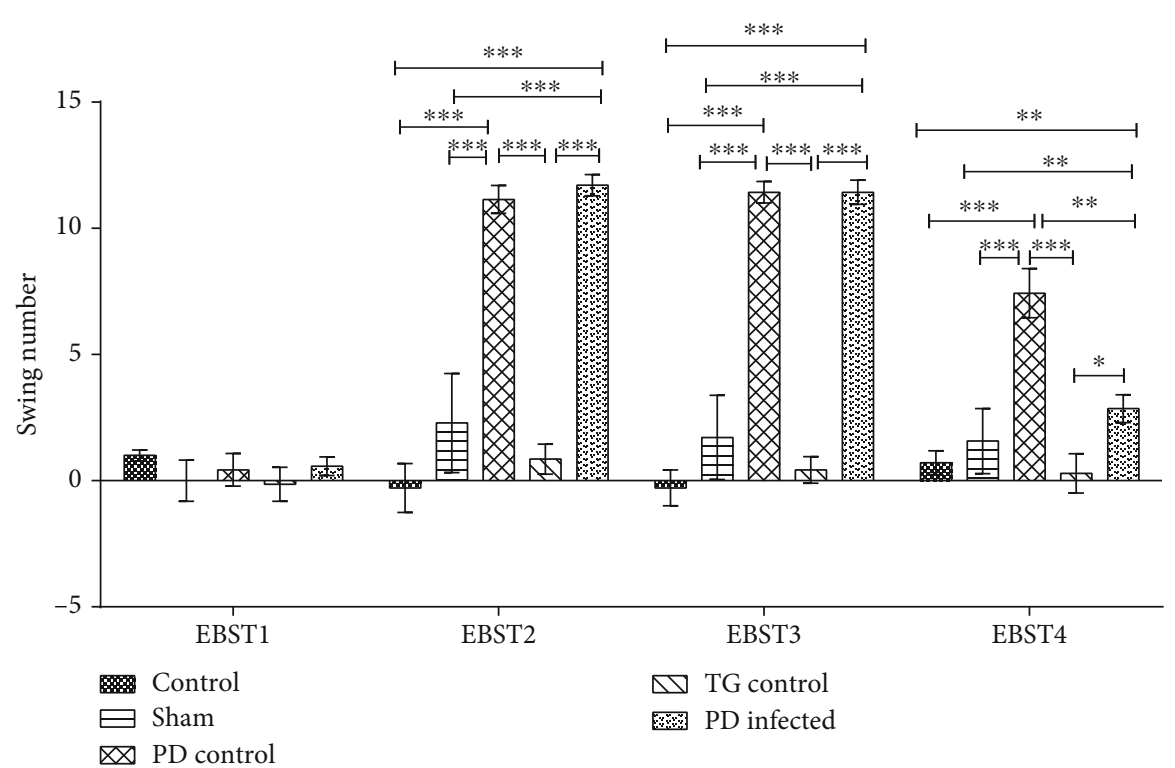

(b)

FIgure 2: Two behavioral tests for PD induction confirmation. (a) Bar test (sec) and (b) EBST test in all groups. ${ }^{*} P<0.05$, ${ }^{* *} P<0.01$, and ${ }^{* * *} P<0.001$ significant differences. Data are shown as means \pm SEM.

fourth step. The TG control group had no significant difference in descent latency time in all steps in comparison with the control and sham groups $(P>0.05)$ (Figure 2(a)).

The elevated body swing test (EBST) confirmed asymmetric behavior representation and success of PD induction. It was performed at four steps of behavioral investigation as EBST1, EBST2, EBST3, and EBST4 (Figure 2(b)). As shown in Figure 2(b), the PD control and PD-infected groups showed a significant $(P<0.001)$ increase in the number of swinging in comparison to the control, sham, and TG control groups in EBST2 and EBST3. In EBST4, the PD-infected group revealed a significant reduction in the biased swing in comparison with the PD control group, whereas it had no significant difference with the control, sham, and TG control groups. However, the PD control group exhibited a sig- nificant increase in the biased swing compared with other groups.

As shown in Figure 3(a), after formalin injection into the hind paw, the PD control group showed a significant increase in the nociceptive scores in the early phase compared to the control and sham $(P<0.05)$ and TG control groups $(P<0.001)$. The PD-infected group had lower formalininduced nociceptive scores in the early phase of the formalin test in comparison with the control and sham $(P<0.05)$ and PD control groups $(P<0.01)$. In the interphase of the formalin test, the PD control group showed a significant $(P<0.01)$ increase in the nociceptive scores in comparison to another groups, but there were no significant differences among the other groups (Figure 3(b)). As illustrated in Figure 3(c), the PD control group had a significant increase in the nociceptive 


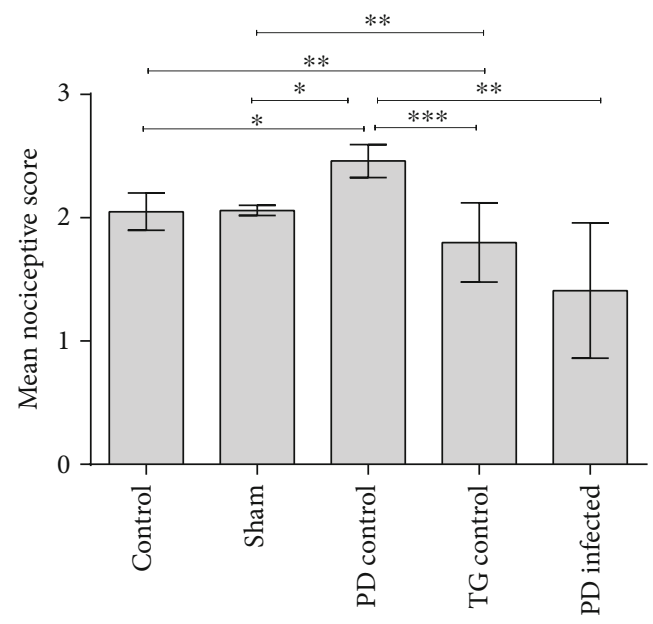

(a)

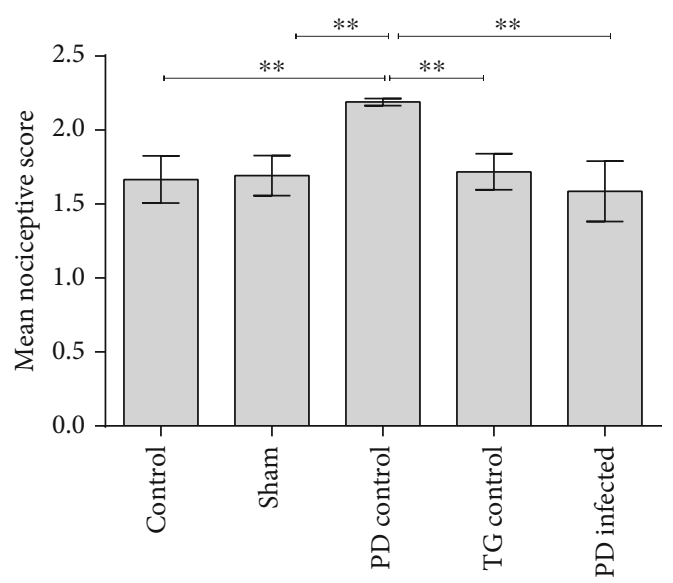

(b)

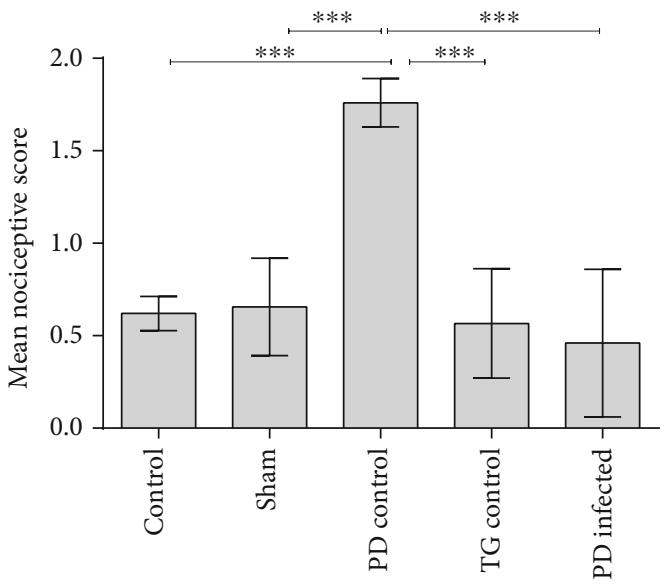

(c)

Figure 3: Nociceptive score in the PD-infected rats. (a) The mean of nociceptive score in the early phase of the formalin test. (b) The mean of the nociceptive score in the interphase of the formalin test. (c) The mean of the nociceptive score in the late phase of the formalin test. ${ }^{*} P<0.05,{ }^{* *} P<0.01$, and ${ }^{* * *} P<0.001$ significant differences. Data are shown as means \pm SEM.

scores in the late phase of formalin test compared to the other groups $(P<0.05)$.

As shown in Figure 4, a significant loss of Nissl-body in the striatal cells was evident in the PD control group compared with other groups $(P<0.001)$. Nevertheless, the PDinfected group significantly recovered from the Nissl-body loss in the striatal cells in comparison with the PD control group $(P<0.001)$. Meanwhile, no significant difference was found between the PD-infected and control, sham, and TG control groups $(P>0.05)$.

According to Figure 5 the number of neuron in striatum was significantly lower compared with other groups $(P<0.01)$. The $\mathrm{PD}$-infected group significantly recovered from the neuron loss in the striatal cells in comparison with the PD control group $(P<0.001)$. Meanwhile, no significant difference was found between the PD-infected and control and sham groups $(P>0.05)$.

Table 1 shows that the percentage of dopamine in striatum neurons of the PD group is lower than that of the control; however, this reduction in the PD control was higher than that of the PD-infected group. Additionally, TG

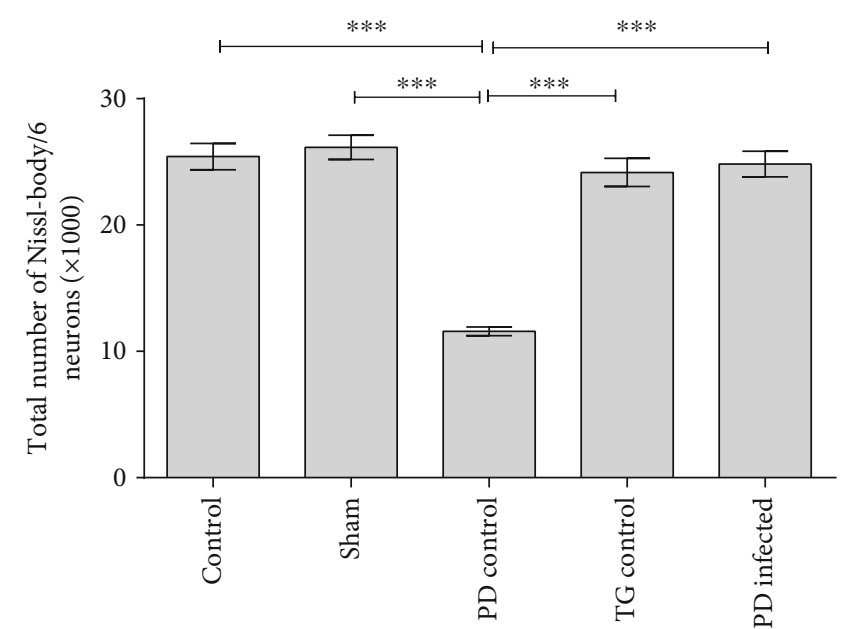

FIGURE 4: The total number of Nissl-bodies in rat striatum cells. ${ }^{* * *} P<0.001$ significant difference. Data are shown as means \pm SEM. 


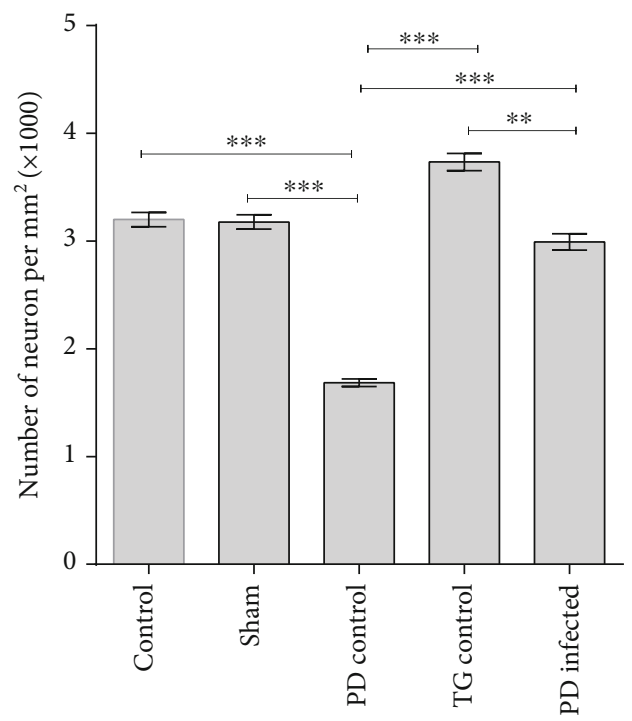

(a)

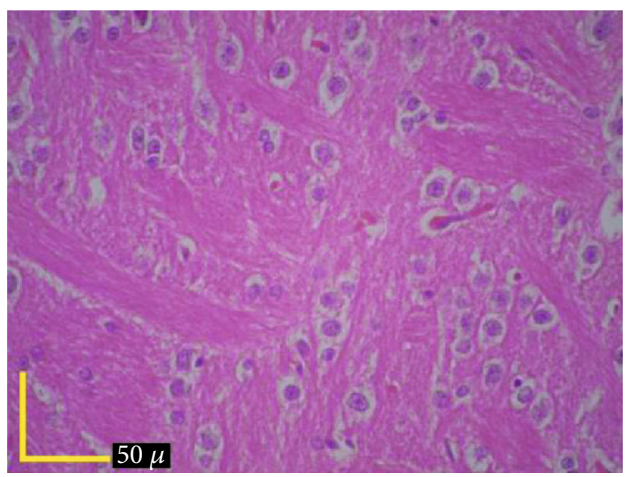

(c)

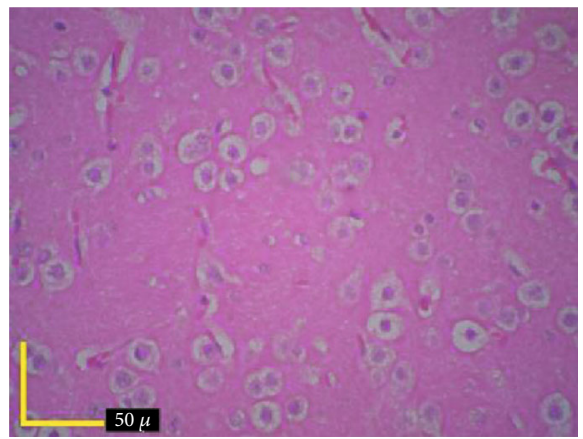

(e)

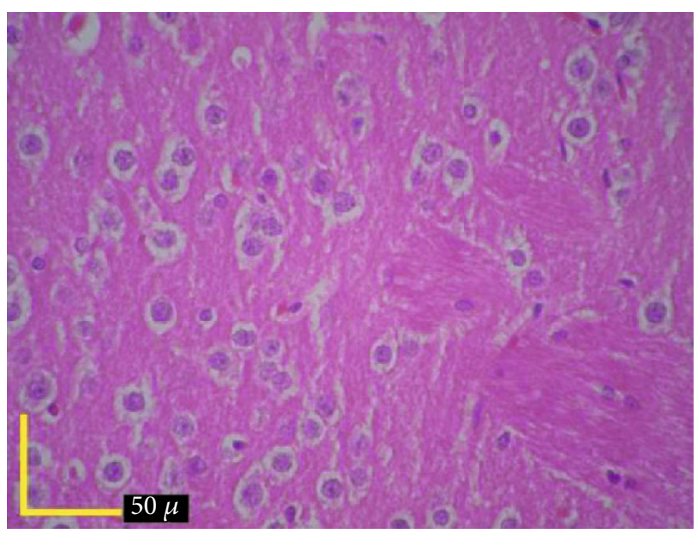

(b)

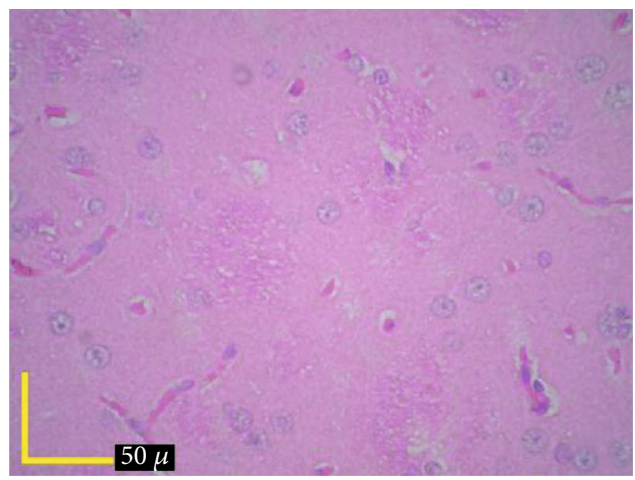

(d)

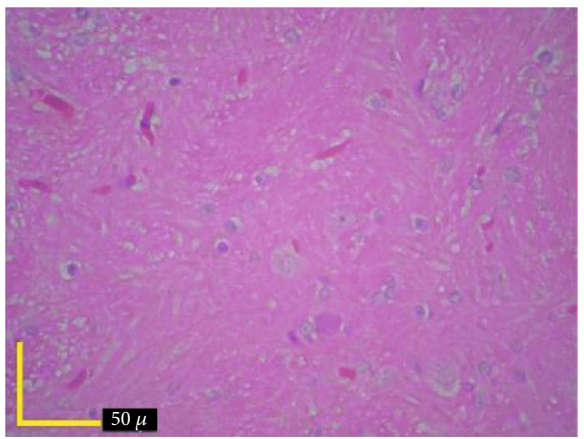

(f)

FIgURE 5: The number of neurons in the striatum (a). Photomicrograph of striatum neuron staining by hematoxylin eosin in the control group (b), sham group (c), PD control group (d), TG control group (e), and PD-infected group (f). ${ }^{* *} P<0.01$; ${ }^{* * *} P<0.001$ significant differences. Data are shown as means \pm SEM.

infection led to an increase in the mean of the dopamine concentration to $25.24 \%$ more than that of the control group.

BDNF of the striatum had a significant reduction $(P<0.001)$ in the PD control group compared to all the other groups (Figure 6(a)). The TG control group showed a significantly higher level of BDNF compared to the PD infected $(P<0.01)$, but PD infected had no significant differences with the control and sham groups $(P>0.05)$.
Figure 6(b) showed that the PD control group significantly decrease the TAC levels compared to the control and sham groups $(P<0.05)$, whereas the PD-infected and TG control groups showed no significant decrease in TAC levels vs. the control and sham groups $(P>0.05)$.

Figure 6(c) showed that the PD control group significantly increased the MDA levels compared to the control and sham groups $(P<0.001)$ whereas the PD-infected and 
TABle 1: Percentage of dopamine reduction relative to the control group.

\begin{tabular}{lc}
\hline Groups & Percentage of dopamine relative to control group \\
\hline Control & $100 \%$ \\
Sham & $97 \%$ \\
PD control & $15.45 \%$ \\
TG control & $+125.24 \%$ \\
PD infected & $63.73 \%$ \\
\hline
\end{tabular}

TG control groups showed no significant increases in MDA levels vs. the control and sham groups $(P>0.05)$.

\section{Discussion}

The aim of the present study was to investigate the effect of the cataleptic and analgesic behaviors of the rat model of PD and how they may be affected by TG infection. Catalepsy is an extrapyramidal dysfunction that is explained as a disability to improve the external abnormal forced positions [24]. In addition to motor symptoms, pain is one of the most troublesome nonmotor symptoms of $\mathrm{PD}$ with a high prevalence that impairs patients' quality of life.

In our experiment, no significant asymmetry and descent latency time were observed among groups in the first step of the behavioral test. Following progressive dopaminergic nigrostriatal degeneration and decrease in the ipsilateral dopamine level, this imbalance resulted in a functional asymmetry [25]. In agreement with the previous studies, the results of this study indicated that PD induction significantly increase the biased swings in the second and third steps of the behavioral test compared with the other groups [20, 25-27]. In the PD-infected group, a significant decrease in the biased swing was observed in the fourth step of the behavioral test compared to the PD control group. TG was able to encode an enzyme with TH activity, a tyrosine to L-DOPA converter, which, in turn, is the rate-limiting step of the dopamine synthesis [28]. As shown in the results, the PD control group caused dopamine depletion by $84.53 \%$ in the striatum tissue. The degree of striatum DA reduction in $\mathrm{PD}$-infected rats was $36.27 \%$; as a result, TG infection has prevented severe dopamine decrease in the striatum. Additionally, the TG control group showed $25.24 \%$ of dopamine amplification in the striatum. In agreement with our finding, an increase for dopamine in the different regions of infected mouse brains including the striatum has been reported [11]. Decreasing dopamine in the lesioned striatum has been known as the primary factor of cataleptic behavior with concomitant descent latency [29]. Induction of PD causes damage to the nigrostriatal dopaminergic neurons, resulting in the abnormal firing of the basal ganglia circuits, manifested as muscle rigidity and catalepsy. Our results were consistent with those of other studies and showed that the descent latency time increased in the 6-OHDA-lesioned rats compared with other groups $[30,31]$. However, PD-infected rats showed a significant decrease in descent latency.

Similar to the previous studies, in our experiment, in the PD control group, dopamine depletion increased formalin- induced pain behaviors in all three phases of the formalin test compared with the other groups $[3,5]$. Hence, it can be concluded that the nigrostriatal dopaminergic system plays an important role in the processing of pain behavior. Previous studies have shown that the intrastriatal injection of dopamine D2 receptor agonists inhibits pain responses [32]. Present results were in agreement with a report that brain dopamine levels increased by TG infection [33]. TG infection improved PD-induced hyperalgesia and rats exhibited less pain behavior compared to the control, sham, and PD control groups in the early phase, interphase, and late phase of the formalin test.

Diminution of the asymmetric behavior following TG infection probably indicates its potency in increasing the striatal dopamine at a level that attenuates motor bias. It has been shown that the increase in the dopaminergic neuron activity and dopamine release could attenuate the catalepsy induced by PD [30]. Striatal medium spiny neurons, in addition to dopaminergic projections from the substantia nigra (inhibitory pathway), receive the glutamatergic input (excitatory pathway) from the motor cortex [34]. Previous research have indicated that the decrease in the function of glutamatergic neurons or NMDA-selective glutamate receptor within the striatum could lead to a reduction in the catalepsy of PD patients [35]. Hama et al. found that NMDA agonist receptors involved in the maintenance of hyperalgesia and NMDA antagonists have analgesic effects on the management of hyperalgesia [36]. Kynurenic acid is an endogenous NMDA-receptor antagonist with antiexcitotoxicity activity in the brain. Schwarcz and Hunter showed that TG infection could increase the synthesis of kynurenic acid in the brain via an immune process [16]. The effectiveness of kynurenic acid in the relief of pain has been confirmed in recent studies [37]. It could be a possible explanation for our observations, where in the fourth step of behavioral test, the PD-infected rats revealed a significant attenuated cataleptic and pain behavior. In the present study, it seems that TG via increasing striatal dopamine and decreasing glutamate through NMDA receptor exerts anticataleptic and analgesic effects on $\mathrm{PD}$ rats.

Meanwhile, in present study, the Nissl-body and neuron distribution in the ipsilateral striatum to the site of 6-OHDA injection have shown significant reduction in the Nissl-body of striatum neurons in the PD control group compared to the other groups; whereas, no significant differences were observed between the PD-infected, control, and sham groups. Therefore, TG could compensate for the decrease in the Nissl-body and neuron numbers induced by PD in the rats' striatum neurons.

Brain-derived neurotrophic factor (BDNF) is a member of the nerve growth factor (NGF) with a critical role in neuronal development, survival, and plasticity that is reduced in PD's brain [38]. Previous studies have demonstrated that BDNF could play a primary role in the protection of dopaminergic neurons against neurotoxins [39]. Present results revealed that TG infection could increase striatal BDNF level in $\mathrm{PD}$-infected rats in comparison with the $\mathrm{PD}$ control rats. Therefore, TG recovered neurons against the neurotoxicity of 6-OHDA, probably due to improving striatal BDNF level. 


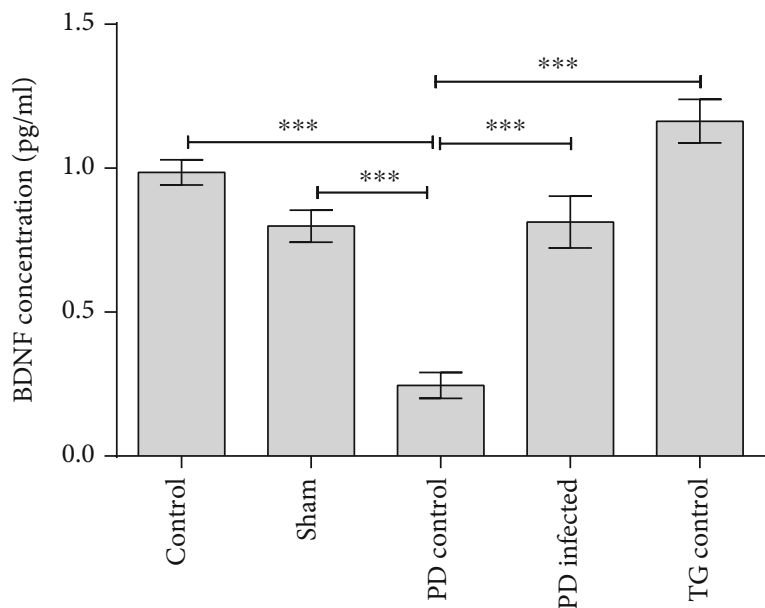

(a)

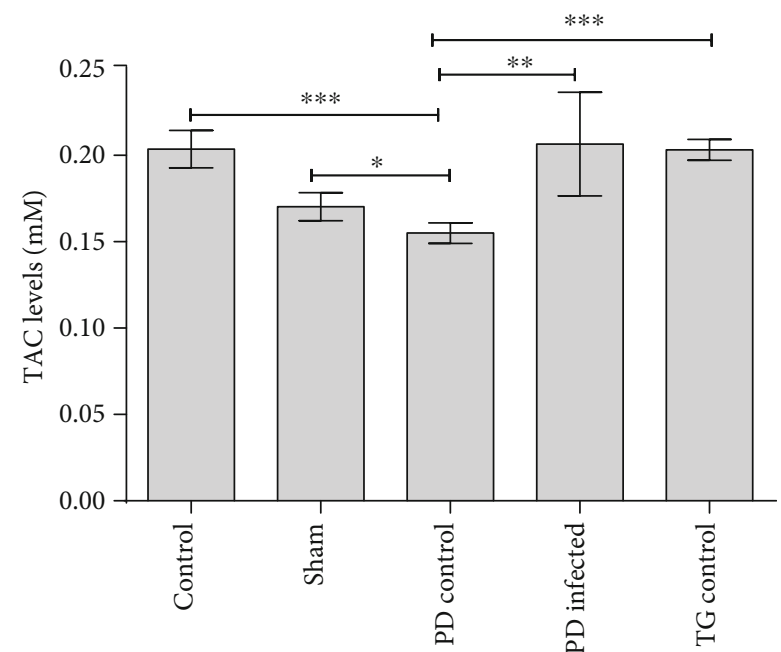

(b)

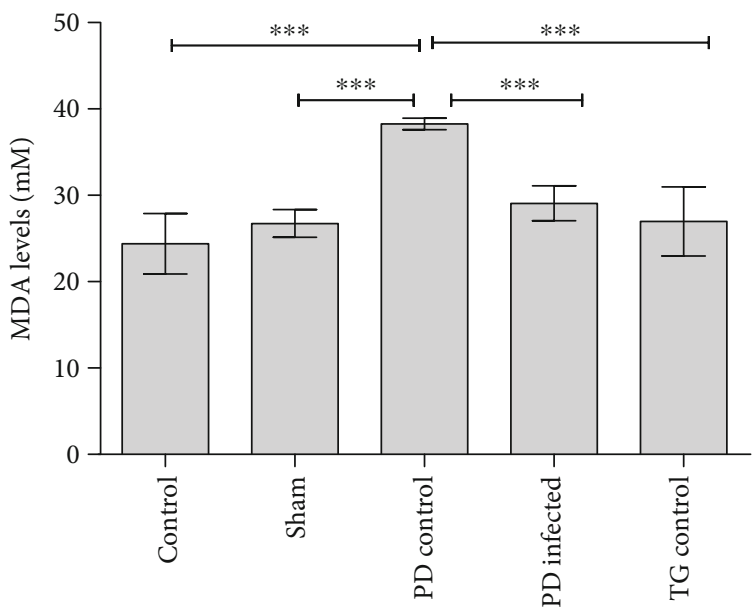

(c)

FIGURE 6: (a) Brain-derived neurotrophic factor (BDNF) protein concentration of striatum tissue. (b) Total antioxidant capacity (TAC) of striatum tissue. (c) Malondialdehyde (MDA) levels of striatum tissue. ${ }^{*} P<0.05,{ }^{* *} P<0.01$, and ${ }^{* * *} P<0.001$ significant differences. Data are shown as means \pm SEM.

Consistent with our results, Xiao et al. reported that TG could increase the BDNF levels in infected mouse striatum tissue. They also demonstrated microRNA- (miR-) 132 high expression in the striatum of infected mice [11]. Transcription of miR-132 in neuronal cells is upregulated by BDNF; it seems that miR-132 mediated the effects of BDNF on the brain [40]. Therefore, miR-132 plays important roles in neurogenesis, synaptic plasticity and neuronal differentiation, neuronal outgrowth, and sprouting [41]. It regulates the differentiation of dopamine neurons from mouse embryonic stem cells [42]. Consequently, it seems that BDNF protects dopaminergic neurons against oxidative stress and prevents cell death [39]. It seems that TG protects cells against oxidative stress via increasing the BDNF levels.

Present findings showed a significant increase in the levels of striatal MDA and decrease in the TAC levels in the PD control group rats compared with the control and sham groups. TG is able to express an antioxidant system, including superoxide dismutase, catalase, at least one peroxiredoxin and complete thioredoxin, and glutathione-based antioxidant systems, that could protect cells against reactive oxygen species (ROS) [43]. An in vitro study by Zhou et al. showed that $T G$ infection caused a significant reduction of NADPH oxidase 4 (Nox4) mRNA and protein level and intracellular reactive oxygen species (ROS) level of the host. These results suggest that Nox4 is the main target for TG in reducing host intracellular ROS level, and PI3K/Akt signaling pathway is responsible for the suppression of Nox4 expression. The results are consistent with our in vivo results in oxidative stress [44].

\section{Conclusion}

Present investigation revealed that TG infection might repair cataleptic, asymmetric, and pain behaviors in the PD control rats by the following ways: (1) enhancing the release of dopamine in the striatum, (2) inhibiting the NMDA-selective glutamate receptor within the striatum by increasing the 
synthesis of kynurenic acid and improving the striatal BDNF level of the rescued neurons against 6-OHDA, and (3) enhancing the TAC levels and decreasing the MDA levels. Further studies are required for a deeper understanding of the proposed pathways responsible for the anticataleptic, antiasymmetric, antinociceptive, and biochemical changes, which are associated with the TG infection in the PD rats. The results of the present study can be used for the development of new drugs or vaccine for the treatment of Parkinson's disease.

\section{Data Availability}

The data used to support the findings of this study are available from the corresponding author upon request.

\section{Ethical Approval}

The animal handling was conducted according to the Ethical Committee for Animal Experiments at Shiraz University.

\section{Conflicts of Interest}

The authors declare that they have no conflicts of interest.

\section{Acknowledgments}

All the people who helped to do this study were included as authors. This study was financially supported by Shiraz University (grant\#: 94GCU4M1755).

\section{References}

[1] P. Redgrave, N. Vautrelle, and J. N. Reynolds, "Functional properties of the basal ganglia's re-entrant loop architecture: selection and reinforcement," Neuroscience, vol. 198, pp. 138-151, 2011.

[2] D. W. Dickson, "Parkinson's disease and parkinsonism: neuropathology," Cold Spring Harbor Perspectives in Medicine, vol. 2, no. 8, 2012.

[3] H. Maegawa, Y. Morimoto, C. Kudo et al., "Neural mechanism underlying hyperalgesic response to orofacial pain in Parkinson's disease model rats," Neuroscience Research, vol. 96, pp. 59-68, 2015.

[4] I. D. Ionov, I. I. Pushinskaya, L. A. Roslavtseva, and N. N. Severtsev, "Brain sites mediating cyclosomatostatin-induced catalepsy in Wistar rats: a specific role for the nigrostriatal system and locus coeruleus," Brain Research, vol. 1691, pp. 26-33, 2018.

[5] M. Dolatshahi, Y. Farbood, A. Sarkaki, S. M. Mansouri, and A. Khodadadi, "Ellagic acid improves hyperalgesia and cognitive deficiency in 6-hydroxidopamine induced rat model of Parkinson's disease," Iranian Journal of Basic Medical Sciences, vol. 18, no. 1, pp. 38-46, 2015.

[6] M. Wang, J. Zhu, Y. Pan et al., "Hydrogen sulfide functions as a neuromodulator to regulate striatal neurotransmission in a mouse model of Parkinson's disease," Journal of Neuroscience Research, vol. 93, no. 3, pp. 487-494, 2015.

[7] W. Zhang, H. An, F. Zhang et al., "Triptolide protects dopaminergic neurons from 6-OHDA lesion in a rat model of Parkin- son's disease," International Journal of Pharmacology, vol. 11, no. 1, pp. 10-18, 2015.

[8] D. E. Hill and J. Dubey, "Toxoplasma gondii," in Foodborne Parasites, Y. Ortega and C. Sterling, Eds., pp. 119-138, Springer, 2018.

[9] N. Mammari, M. A. Halabi, S. Yaacoub, H. Chlala, M. L. Darde, and B. Courtioux, "Toxoplasma gondii modulates the host cell responses: an overview of apoptosis pathways," BioMed Research International, vol. 2019, Article ID 6152489, 10 pages, 2019.

[10] M. Ding, C. Clayton, and D. Soldati, “Toxoplasma gondii catalase: are there peroxisomes in toxoplasma?," Journal of Cell Science, vol. 113, pp. 2409-2419, 2000.

[11] J. Xiao, Y. Li, E. Prandovszky et al., "MicroRNA-132 dysregulation in Toxoplasma gondii infection has implications for dopamine signaling pathway," Neuroscience, vol. 268, pp. 128-138, 2014.

[12] E. Prandovszky, E. Gaskell, H. Martin, J. P. Dubey, J. P. Webster, and G. A. McConkey, "The neurotropic parasite Toxoplasma gondii increases dopamine metabolism," PLoS One, vol. 6, no. 9, article e23866, 2011.

[13] L. Kim and E. Y. Denkers, "Toxoplasma gondii triggers Gidependent PI 3-kinase signaling required for inhibition of host cell apoptosis," Journal of Cell Science, vol. 119, no. 10, pp. 2119-2126, 2006.

[14] G. A. McConkey, H. L. Martin, G. C. Bristow, and J. P. Webster, "Toxoplasma gondii infection and behaviour-location, location, location?," Journal of Experimental Biology, vol. 216, no. 1, pp. 113-119, 2012.

[15] L. Torres, S. A. Robinson, D. G. Kim, A. Yan, T. A. Cleland, and M. S. Bynoe, "Toxoplasma gondii alters NMDAR signaling and induces signs of Alzheimer's disease in wild-type, C57BL/6 mice," Journal of Neuroinflammation, vol. 15, no. 1, p. 57, 2018.

[16] R. Schwarcz and C. A. Hunter, "Toxoplasma gondii and schizophrenia: linkage through astrocyte-derived kynurenic acid?," Schizophrenia Bulletin, vol. 33, no. 3, pp. 652-653, 2007.

[17] M. D. Neely, D. E. Schmidt, and A. Y. Deutch, "Cortical regulation of dopamine depletion-induced dendritic spine loss in striatal medium spiny neurons," Neuroscience, vol. 149, no. 2, pp. 457-464, 2007.

[18] N. A. Moore, A. Blackman, S. Awere, and J. D. Leander, "NMDA receptor antagonists inhibit catalepsy induced by either dopamine D1 or D2 receptor antagonists," European Journal of Pharmacology, vol. 237, no. 1, pp. 1-7, 1993.

[19] K. Hattori, S. Uchino, T. Isosaka et al., "Fyn is required for haloperidol-induced catalepsy in mice," Journal of Biological Chemistry, vol. 281, no. 11, pp. 7129-7135, 2006.

[20] M. Roghani, G. Behzadi, and T. Baluchnejadmojarad, "Efficacy of elevated body swing test in the early model of Parkinson's disease in rat," Physiology \& Behavior, vol. 76, no. 4-5, pp. 507-510, 2002.

[21] N. Xiong, J. Xiong, G. Khare et al., "Edaravone guards dopamine neurons in a rotenone model for Parkinson's disease," PLoS One, vol. 6, no. 6, article e20677, 2011.

[22] M. J. Morgan and K. B. Franklin, “6-Hydroxydopamine lesions of the ventral tegmentum abolish D-amphetamine and morphine analgesia in the formalin test but not in the tail flick test," Brain Research, vol. 519, no. 1-2, pp. 144-149, 1990.

[23] M. V. Mabandla, M. Nyoka, and W. M. U. Daniels, "Early use of oleanolic acid provides protection against 6-hydroxydopamine 
induced dopamine neurodegeneration," Brain Research, vol. 1622, pp. 64-71, 2015.

[24] L. A. Baez, N. K. Eskridge, and R. Schein, "Postnatal development of dopaminergic and cholinergic catalepsy in the rat," European Journal of Pharmacology, vol. 36, no. 1, pp. 155$162,1976$.

[25] R. K. Schwarting and J. P. Huston, "Behavioral and neurochemical dynamics of neurotoxic meso-striatal dopamine lesions," Neurotoxicology, vol. 18, no. 3, pp. 689-708, 1997.

[26] C. V. Borlongan and P. R. Sanberg, "Elevated body swing test: a new behavioral parameter for rats with 6-hydroxydopamineinduced hemiparkinsonism," Journal of Neuroscience, vol. 15, no. 7, pp. 5372-5378, 1995.

[27] H. Yuan, S. Sarre, G. Ebinger, and Y. Michotte, "Histological, behavioural and neurochemical evaluation of medial forebrain bundle and striatal 6-OHDA lesions as rat models of Parkinson's disease," Journal of Neuroscience Methods, vol. 144, no. 1, pp. 35-45, 2005.

[28] H. L. Martin, I. Alsaady, G. Howell et al., "Effect of parasitic infection on dopamine biosynthesis in dopaminergic cells," Neuroscience, vol. 306, pp. 50-62, 2015.

[29] A. Klein and W. J. Schmidt, "Catalepsy intensifies contextdependently irrespective of whether it is induced by intermittent or chronic dopamine deficiency," Behavioural Pharmacology, vol. 14, no. 1, pp. 49-53, 2003.

[30] A. M. Nayebi, S. R. Rad, M. Saberian, S. Azimzadeh, and M. Samini, "Buspirone improves 6-hydroxydopamineinduced catalepsy through stimulation of nigral 5-HT(1A) receptors in rats," Pharmacological Reports, vol. 62, no. 2, pp. 258-264, 2010.

[31] S. K. Prajapati, D. Garabadu, and S. Krishnamurthy, "Coenzyme Q10 prevents mitochondrial dysfunction and facilitates pharmacological activity of atorvastatin in 6-OHDA induced dopaminergic toxicity in rats," Neurotoxicity Research, vol. 31, no. 4, pp. 478-492, 2017.

[32] N. Hagelberg, I. K. Martikainen, H. Mansikka et al., "Dopamine D2 receptor binding in the human brain is associated with the response to painful stimulation and pain modulatory capacity," Pain, vol. 99, no. 1-2, pp. 273-279, 2002.

[33] J. Gatkowska, M. Wieczorek, B. Dziadek, K. Dzitko, and H. Dlugonska, "Sex-dependent neurotransmitter level changes in brains of Toxoplasma gondii infected mice," Experimental Parasitology, vol. 133, no. 1, pp. 1-7, 2013.

[34] D. J. Surmeier, J. Ding, M. Day, Z. Wang, and W. Shen, "D1 and D2 dopamine-receptor modulation of striatal glutamatergic signaling in striatal medium spiny neurons," Trends in Neurosciences, vol. 30, no. 5, pp. 228-235, 2007.

[35] J. T. Greenamyre and C. F. O'Brien, "N-methyl-D-aspartate antagonists in the treatment of Parkinson's disease," Archives of Neurology, vol. 48, no. 9, pp. 977-981, 1991.

[36] A. Hama, J. Woon Lee, and J. Sagen, "Differential efficacy of intrathecal NMDA receptor antagonists on inflammatory mechanical and thermal hyperalgesia in rats," European Journal of Pharmacology, vol. 459, no. 1, pp. 49-58, 2003.

[37] G. Veres, A. Fejes-Szabó, D. Zádori et al., “A comparative assessment of two kynurenic acid analogs in the formalin model of trigeminal activation: a behavioral, immunohistochemical and pharmacokinetic study," Journal of Neural Transmission, vol. 124, no. 1, pp. 99-112, 2017.

[38] K. Parain, M. G. Murer, Q. Yan et al., "Reduced expression of brain-derived neurotrophic factor protein in Parkinson's dis- ease substantia nigra," Neuroreport, vol. 10, no. 3, pp. 557561, 1999.

[39] M. B. Spina, S. P. Squinto, J. Miller, R. M. Lindsay, and C. Hyman, "Brain-derived neurotrophic factor protects dopamine neurons against 6-hydroxydopamine and N-methyl-4phenylpyridinium ion toxicity: involvement of the glutathione system," Journal of Neurochemistry, vol. 59, no. 1, pp. 99-106, 1992.

[40] J. Remenyi, C. J. Hunter, C. Cole et al., "Regulation of the miR212/132 locus by MSK1 and CREB in response to neurotrophins," Biochemical Journal, vol. 428, no. 2, pp. 281-291, 2010.

[41] S. Impey, M. Davare, A. Lasiek et al., "An activity-induced microRNA controls dendritic spine formation by regulating Rac1-PAK signaling," Molecular and Cellular Neuroscience, vol. 43, no. 1, pp. 146-156, 2010.

[42] D. Yang, T. Li, Y. Wang et al., "miR-132 regulates the differentiation of dopamine neurons by directly targeting Nurr1 expression," Journal of Cell Science, vol. 125, no. 7, pp. 1673$1682,2012$.

[43] E. S. Son, K. J. Song, J. C. Shin, and H. W. Nam, "Molecular cloning and characterization of peroxiredoxin from Toxoplasma gondii," The Korean Journal of Parasitology, vol. 39, no. 2, pp. 133-141, 2001.

[44] W. Zhou, J. H. Quan, Y. H. Lee, D. W. Shin, and G. H. Cha, "Toxoplasma gondii proliferation require down-regulation of host Nox4 expression via activation of PI3 kinase/Akt signaling pathway," PLoS One, vol. 8, no. 6, article e66306, 2013. 